Original Article

\title{
The effects of a multisensory dynamic balance training on the thickness of lower limb muscles in ultrasonography in children with spastic diplegic cerebral palsy
}

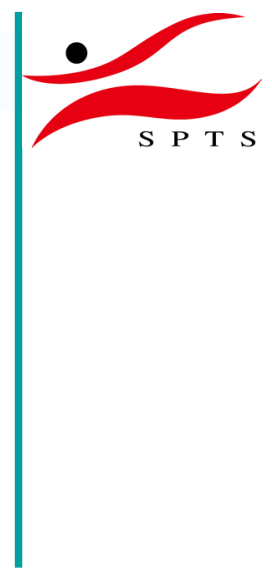

\author{
Seung-min Nam, PT, MSc ${ }^{1)}$, Won-hyo Kim, PT, MSc${ }^{1)}$, Chang-Kyo Yun, PT, MSc ${ }^{1)^{*}}$ \\ 1) Department of Physical Therapy, College of Rehabilitation Science, Graduate School, \\ Daegu University: 15 Jilyang, Gyeongsan-si, Kyeongbuk 712-714, Republic of Korea
}

\begin{abstract}
Purpose] This study aimed to investigate the effects of multisensory dynamic balance training on muscles thickness such as rectus femoris, anterior tibialis, medial gastrocnemius, lateral gastrocnemius in children with spastic diplegic cerebral palsy by using ultrasonography. [Subjects and Methods] Fifteen children diagnosed with spastic diplegic cerebral palsy were divided randomly into the balance training group and control group. The experimental group only received a multisensory dynamic balance training, while the control group performed general physiotherapy focused balance and muscle strengthening exercise based Neurodevelopmental treatment. Both groups had a therapy session for 30 minutes per day, three times a week for six weeks. The ultrasonographic muscle thickness were obtained in order to compare and analyze muscle thickness before and after in each group. [Result] The experimental group had significant increases in muscle thickness in the rectus femoris, tibialis anterior, medial gastrocnemius and lateral gastrocnemius muscles. The control group had significant increases in muscle thickness in the tibialis anterior. The test results of the rectus femoris, medial gastrocnemius and lateral gastrocnemius muscle thickness values between the groups showed significant differences. [Conclusion] In conclusion, a multisensory dynamic balance training can be recommended as a treatment method for patients with spastic diplegic cerebral palsy. Key words: Balance control training, Spastic diplegic cerebral palsy, Muscle thickness
\end{abstract}

(This article was submitted Nov. 3, 2016, and was accepted Jan. 24, 2017)

\section{INTRODUCTION}

Cerebral palsy (CP) is defined as non-progressive brain damage that occurred when the brain was immature. Children with $\mathrm{CP}$ experience stiffness, contractures, weakening muscle strength, sensory defects, and difficulties in balancing and motion control, which can lead to functional disabilities ${ }^{1)}$. In the type of CP, spastic diplegic cerebral palsy affects stiffness of the limbs on both sides anatomically and kinematically, and affects the lower extremities more than the upper extremities ${ }^{2)}$. Children with spastic diplegic cerebral palsy experience decrease of muscle strength in their lower extremities due to reduced functional activities for a long time, which is followed by reduced motor ability ${ }^{3}$. In particular, the severe decrease of muscle strength occurs at the knee extensors, dorsiflexors, and plantar flexors of children with spastic diplegic cerebral palsy ${ }^{4,5)}$.

In previous studies, a number of training methods such as muscle strengthening training, isotonic muscle strengthening training, and isokinetic muscle strength training using resistance have been reported in order to improve muscle strength in lower extremities of children with spastic diplegic cerebral palsy ${ }^{6}$. However, it is difficult for children with CP to continue monotonous muscle strengthening training because monotonous training methods are difficult to keep interest and motivate in children. On the other hand, a multisensory dynamic balance training can overcome the monotonous training methods by

*Corresponding author. Chang-kyo Yun (E-mail: puhaha1116@naver.com)

(C2017 The Society of Physical Therapy Science. Published by IPEC Inc.

This is an open-access article distributed under the terms of the Creative Commons Attribution Non-Commercial No Derivatives (by-nc-nd) License $<$ https://creativecommons.org/licenses/by-nc-nd/4.0/>. 
Table 1. General characteristics of subjects

\begin{tabular}{|c|c|c|c|}
\hline & & Experimental group & Control group \\
\hline \multirow[t]{2}{*}{ Gender } & M & 6 & 4 \\
\hline & $\mathrm{F}$ & 2 & 3 \\
\hline \multicolumn{2}{|c|}{ Age (years) } & $14.88 \pm 3.80$ & $14.14 \pm 3.67$ \\
\hline \multicolumn{2}{|c|}{ Height $(\mathrm{cm})$} & $153.75 \pm 17.98$ & $154.29 \pm 20.68$ \\
\hline \multicolumn{2}{|c|}{ Weight (kg) } & $48.75 \pm 16.07$ & $46.29 \pm 12.94$ \\
\hline \multicolumn{2}{|c|}{ GMFCS (score) } & $1.75 \pm 0.71$ & $1.57 \pm 0.53$ \\
\hline
\end{tabular}

allowing the children to perform active exercise while watching the monitor ${ }^{7}$.

Furthermore, most muscle evaluation methods conducted in South Korea's clinical fields are not based on objective analysis methods, but rather employ a scoring method after observing the performance of patients ${ }^{8)}$. To overcome this subjective scoring method, ultrasonography can be used to evaluate muscle functions. Ultrasonography produces ultrasound images, which are compared and analyzed, thereby influencing treatment mechanisms and methods in clinical fields ${ }^{9)}$. Thus, the purpose of the present study was to investigate the effects of a multisensory dynamic balance training on ultrasonographic muscle thickness such as the rectus femoris, tibialis anterior, medial gastrocnemius, and lateral gastrocnemius in children with spastic diplegic cerebral palsy.

\section{SUBJECTS AND METHODS}

This study was conducted from July to October 2015 after receiving approval from the Bioethics Committee of Daegu University. All study participants and guardians were informed of the study's purpose and procedures methods and gave consent for participation. The participants were 15 children diagnosed with spastic diplegic cerebral palsy who were outpatients of the D Hospital in Daegu (5 female and 10 male children). The selection criteria were children with spastic diplegic cerebral palsy who belonged to GMFCSI-III and who had a level of stiffness that was less than 2 on the MAS, children without visual and auditory deficits, and children who had not had orthopedic surgery or botuline toxin treatment in the last six months (Table 1).

In this study, the subjects were divided into each group by using randomized control traial with a blinded evaluator. An experimental group consisting of eight subjects who conducted only a multisensory dynamic balance training and control group consisting of seven subjects who conducted only general physiotherapy that focused on dynamic balancing and muscle strengthening exercises based on Neurodevelopmental treatment. The intervention period was six weeks for both groups and the exercises were conducted 30 minutes per day, three times per week. An examination was conducted prior to the intervention and a post examination was conducted after the 6-week intervention.

A Bal Pro (Man \& Tel Co., Korea) was used as the dynamic balance trainer and knee joint movements were applied. The balance trainer consists of a screen, a pressure sensor that can sense the horizontal movements of weight loads on the foothold, a tilt sensor that can perceive the angle of the knee joint, and a main body that contains programs that process the information perceived by the tilt sensor. The pressure sensor on the foothold can sense the body weight centers of the patients to help them move horizontally using a finger-shaped cursor on the screen during the exercise as the body weight moves to the right or left. A tilt sensor detects the angle of flexion and extension of the knee joints. The training involves using the cursor to touch various pieces of fruit arranged randomly on a tree on the screen ${ }^{10)}$.

An Accuvix V10 (Samsung Medicine Co., South Korea) and $10 \mathrm{MHz}$ linear probe were used as the ultrasonic measuring system for the examinations. All measurements were conducted by a physical therapist who was blinded to this study's purpose and which group the subjects belonged. The thicknesses of the rectus femoris, which is a knee extensor group; the tibialis anterior, which is an ankle joint dorsiflexor group; and the medial and lateral gastrocnemius, which are plantar flexors; were measured. The thicknesses of the muscles on the right side were measured for all subjects. All measurements were conducted while the subjects were in a comfortable position without muscle contraction ${ }^{11,12)}$.

Data analyses were performed using the SPSS program version 18.0. A paired t-test was performed for the intragroup comparison, and an independent t-test was done for the intergroup comparison. The significance level was set to 0.05 .

\section{RESULTS}

The measurement results of the muscle thickness in the RF, mGCM and 1GCM muscle showed that the experimental group had a significant increase in the thickness values before and after the exercise $(\mathrm{p}<0.05)$, and the control group had no significant increase in the thickness values before and after the exercise $(p>0.05)$. The measurement results of the muscle thickness in the TA showed that both the experimental and control groups had a significant increase in the thickness values before and after the exercise $(\mathrm{p}<0.05)$. The test results of the RF, mGCM and IGCM muscle thickness values between the 
Table 2. Comparison of muscle thickness for each group at pre-test and post-test

\begin{tabular}{lcccc}
\hline & \multicolumn{2}{c}{ Experimental group } & \multicolumn{2}{c}{ Control group } \\
\cline { 2 - 5 } & pre-test $^{\mathrm{a}}$ & post-test $^{\mathrm{a}}$ & pre-test $^{\mathrm{a}}$ & post-test $^{\mathrm{a}}$ \\
\hline Rectus femoris (cm) & $1.96 \pm 0.43$ & $2.11 \pm 0.43^{* *}$ & $1.57 \pm 0.51$ & $1.59 \pm 0.49$ \\
Tibialis anterior (cm) & $1.61 \pm 0.42$ & $1.80 \pm 0.45^{*}$ & $1.47 \pm 0.36$ & $1.58 \pm 0.35^{*}$ \\
Medial gastrocnemius (cm) & $1.16 \pm 0.26$ & $1.28 \pm 0.26^{* *}$ & $1.08 \pm 0.34$ & $1.04 \pm 0.14$ \\
Lateral gastrocnemius (cm) & $1.09 \pm 0.21$ & $1.16 \pm 0.17^{* *}$ & $0.90 \pm 0.21$ & $0.95 \pm 0.21$ \\
\hline
\end{tabular}

${ }^{\mathrm{a}}$ Mean $\pm \mathrm{SE}$.

*Significant difference within the group $(\mathrm{p}<0.05),{ }^{*}$ Significant difference between the groups $(\mathrm{p}<0.05)$

groups showed a significant difference $(\mathrm{p}>0.05)$ (Table 2).

\section{DISCUSSION}

Previous studies have reported a high correlation between muscle strength and muscle thickness. Thus, the present study aimed to determine the effects of a multisensory dynamic balance training on muscle thickness in the lower extremities of children with spastic diplegic cerebral palsy and whether the multisensory dynamic balance training can be proposed as a new treatment method. The study results showed that the multisensory dynamic balance training significantly and statistically increased the thicknesses of all four muscles (rectus femoris, tibialis anterior, medial gastrocnemius, and lateral gastrocnemius). This result indicated that balance training that applies knee joint motions stimulates a proprioceptor, thereby increasing sensory nerve signaling from the pressure receptors of ankle joints, which led to an increase in the motion control of the surrounding muscles of the ankle and knee joints ${ }^{13)}$. Moreover, the comparison results of muscle thickness between the experimental and control groups in this study showed a significant difference in the thickness of the rectus femoris, medial gastrocnemius, and lateral gastrocnemius after the training. This result indicated that the rectus femoris played a role in the extension of the knee joint as well as the flexion of the hip joint while gastrocnemius played a role in the plantar flexion of the ankle joints as well as the flexion of the knee joints. These muscles are two-joint muscles and they were considered more effective to increase in the muscle thickness of rectus femoris, medial gastrocnemius, and lateral gastrocnemius in the multisensory dynamic balance training of the experimental group than in the control group due to the motions of flexion and the extension of the knee joints. Moreover, gastrocnemius and rectus femoris were positioned in an extended location in a relative sense due to the feet located at the posterior of the knee during knee joint flexion. Here, the extended muscle was combined with the balance training equipment, thereby improving the gastrocnemius and rectus femoris more significantly than the other muscles due to the relationship between length and tension ${ }^{14)}$. As a result, children with spastic diplegic cerebral palsy should focus on the functions of all muscles and joints in the lower extremities rather than focusing on each individual muscle and joint. The limitation in this study include a lack of functional outcome measurement, a small subject size and a lack of accessibility to the balance training machine. Through the study results, knee joint motion-applied balance training was found to be effective for improving the musculoskeletal structure of children with spastic diplegic cerebral palsy.

\section{Conflict of interest}

The authors have no conflict of interest to declare.

\section{REFERENCES}

1) Nelson KB: Can we prevent cerebral palsy? N Engl J Med, 2003, 349: 1765-1769. [Medline] [CrossRef]

2) Jones ET, Knapp DR: Assessment and management of the lower extremity in cerebral palsy. Orthop Clin North Am, 1987, 18: 725-738. [Medline]

3) Damiano DL, Abel MF: Functional outcomes of strength training in spastic cerebral palsy. Arch Phys Med Rehabil, 1998, 79: 119-125. [Medline] [CrossRef]

4) Taylor NF, Dodd KJ, Larkin H: Adults with cerebral palsy benefit from participating in a strength training programme at a community gymnasium. Disabil Rehabil, 2004, 26: 1128-1134. [Medline] [CrossRef]

5) Rodda J, Graham HK: Classification of gait patterns in spastic hemiplegia and spastic diplegia: a basis for a management algorithm. Eur J Neurol, 2001, 8: 98-108. [Medline] [CrossRef]

6) Fowler EG, Ho TW, Nwigwe AI, et al.: The effect of quadriceps femoris muscle strengthening exercises on spasticity in children with cerebral palsy. Phys Ther, 2001, 81: 1215-1223. [Medline]

7) Lai CH, Peng CW, Chen YL, et al.: Effects of interactive video-game based system exercise on the balance of the elderly. Gait Posture, 2013 , 37: 511-515. [Medline] [CrossRef]

8) Steenbergen B, van Thiel E, Hulstijn W, et al.: The coordination of reaching and grasping in spastic hemiparesis. Hum Mov Sci, 2000, 19: 75-105. [CrossRef]

9) Li L, Tong KY, Hu X: The effect of poststroke impairments on brachialis muscle architecture as measured by ultrasound. Arch Phys Med Rehabil, 2007, 88: 243-250. [Medline] [CrossRef] 
10) Nam SM, Kim WB, Yun CK: Effects of balance training by knee joint motions on muscle activity in adult men with functional ankle instability. J Phys Ther Sci, 2016, 28: 1629-1632. [Medline] [CrossRef]

11) Moreau NG, Teefey SA, Damiano DL: In vivo muscle architecture and size of the rectus femoris and vastus lateralis in children and adolescents with cerebral palsy. Dev Med Child Neurol, 2009, 51: 800-806. [Medline] [CrossRef]

12) Yun CK, Kim WH, Kim SG: Partial correlation between lower muscle thickness, 10-meter walk test, and the timed up \& go test in children with spastic cerebral palsy. J Phys Ther Sci, 2016, 28: 1611-1613. [Medline] [CrossRef]

13) Silva A, Sousa AS, Pinheiro R, et al.: Activation timing of soleus and tibialis anterior muscles during sit-to-stand and stand-to-sit in post-stroke vs. healthy subjects. Somatosens Mot Res, 2013, 30: 48-55. [Medline] [CrossRef]

14) Beltman JG, Sargeant AJ, van Mechelen W, et al.: Voluntary activation level and muscle fiber recruitment of human quadriceps during lengthening contractions. J Appl Physiol 1985, 2004, 97: 619-626. [Medline] [CrossRef] 